\title{
A SCORZA-DRAGONI APPROACH TO DIRICHLET PROBLEM WITH AN UPPER-CARATHÉODORY RIGHT-HAND SIDE
}

\author{
Martina PAVlaČKová
}

\begin{abstract}
In this paper, the existence and localization result will be proven for multivalued vector Dirichlet problem with an upperCarathéodory right-hand side by using bound sets approach. Since ScorzaDragoni type technique will be furthermore applied, the conditions for bounding functions can be required directly on the boundaries of bound sets and not at some vicinity of them.
\end{abstract}

\section{Introduction}

Boundary value problems (b.v.p.) for second-order differential inclusions have been studied for many years (see, e.g. [1], [5], [6], [9]-[11], [13]) since to their applications in several areas, such as physics, control theory or mathematical economics. In mentioned papers, various methods (like an upper and lower solutions technique, topological transformations, fixed point technique or tube solution method) were applied for obtaining the existence results. In this paper, except for the existence of a solution, also its localization is studied for multivalued vector Dirichlet problem. More concretely, let us consider the Dirichlet multivalued problem

$$
\left\{\begin{array}{l}
\ddot{x}(t) \in F(t, x(t), \dot{x}(t)), \quad \text { for a.a. } t \in[0, T], \\
x(T)=x(0)=\mathbf{0}
\end{array}\right.
$$

2010 Mathematics Subject Classification. 34A60, 34B15.

Key words and phrases. Dirichlet problem, upper-Carathéodory differential inclusions, localization of a solution, strictly localized bounding functions, Scorza-Dragoni technique. 
where $F:[0, T] \times \mathbb{R}^{n} \times \mathbb{R}^{n} \multimap \mathbb{R}^{n}$ is an upper-Carathéodory multivalued mapping, together with the localization condition

$$
x(t) \in K, \quad \text { for all } t \in[0, T],
$$

where $K \subset \mathbb{R}^{n}$ is given open bounded set containing the null vector $\mathbf{0}$.

Let us note that the notion of a solution will be understood in the strong sense, i.e. by a solution of problem (1.1)-(1.2) we shall mean a function $x:[0, T]$ $\rightarrow \mathbb{R}^{n}$ with absolutely continuous first derivative satisfying (1.1)-(1.2).

Dirichlet viability problem (1.1)-(1.2) was already studied in [7], [12]. In [7], the multivalued mapping $F$ was globally u.s.c. and conditions for bounding functions were imposed directly on boundaries of bound sets, while in [12], $F$ was an upper-Carathéodory multivalued mapping but the conditions concerning bounding functions were imposed at some vicinity of the boundaries of bounds sets. Since the Scorza-Dragoni type technique is applied in the present paper, conditions for bounding functions are imposed directly on boundaries of bound sets also in the case of upper-Carathéodory right-hand side. The obtained result is at the end of the paper illustrated by the vector dry friction problem.

\section{Preliminaries}

Let us start with notations we use in the paper. If $(X, d)$ is a metric space and $A \subset X$, by $\bar{A}$, Int $A$, and $\partial A$, we mean the closure, the interior, and the boundary of $A$, respectively. For a subset $A \subset X$ and $\varepsilon>0$, we define the set $N_{\varepsilon}(A):=\{x \in X \mid$ there exists $a \in A: d(x, a)<\varepsilon\}$, i.e. $N_{\varepsilon}(A)$ is an open neighbourhood of the set $A$ in $X$.

For a given compact real interval $J$, we denote by $C\left(J, \mathbb{R}^{n}\right)\left(\right.$ by $\left.C^{1}\left(J, \mathbb{R}^{n}\right)\right)$ the set of all functions $x: J \rightarrow \mathbb{R}^{n}$ which are continuous (have continuous first derivatives) on $J$. $\operatorname{By~} \operatorname{AC}^{1}\left(J, \mathbb{R}^{n}\right)$, we shall mean the set of all functions $x: J \rightarrow \mathbb{R}^{n}$ with absolutely continuous first derivatives on $J$.

We also need following definitions and notions from multivalued theory in the sequel. We say that $\mathrm{F}$ is a multivalued mapping from $X$ to $Y$ (written $F: X \multimap Y$ ) if, for every $x \in X$, a nonempty subset $F(x)$ of $Y$ is given. A multivalued mapping $F: X \multimap Y$ is called upper semi-continuous (shortly, u.s.c.) if, for each open set $U \subset Y$, the set $\{x \in X \mid F(x) \subset U\}$ is open in $X$.

Let $Y$ be a metric space and $(\Omega, \mathcal{U}, \mu)$ be a measurable space, i.e. a nonempty set $\Omega$ equipped with a suitable $\sigma$-algebra $\mathcal{U}$ of its subsets and a countably additive measure $\mu$ on $\mathcal{U}$. A multivalued mapping $F: \Omega \multimap Y$ is called measurable if $\{\omega \in \Omega \mid F(\omega) \subset V\} \in \mathcal{U}$, for each open set $V \subset Y$. In the sequel, the symbol $\mu$ will exclusively denote the Lebesgue measure on $\mathbb{R}$.

We say that mapping $F: J \times \mathbb{R}^{m} \multimap \mathbb{R}^{n}$, where $J \subset \mathbb{R}$ is a compact interval, is an upper-Carathéodory mapping if the map $F(\cdot, x): J \multimap \mathbb{R}^{n}$ is measurable, 
for all $x \in \mathbb{R}^{m}$, the map $F(t, \cdot): \mathbb{R}^{m} \multimap \mathbb{R}^{n}$ is u.s.c., for almost all $t \in J$, and the set $F(t, x)$ is compact and convex, for all $(t, x) \in J \times \mathbb{R}^{m}$.

In the proof of the main result, the following slight modification of ScorzaDragoni type technique for multivalued mappings will be employed.

Proposition 2.1 (cf. e.g. [4, Proposition 8]). Let $X \subset \mathbb{R}^{m}$ be compact and let $F:[a, b] \times X \multimap \mathbb{R}^{n}$ be an upper-Carathéodory mapping. Then there exists a multivalued mapping $F_{0}:[a, b] \times X \multimap \mathbb{R}^{n} \cup\{\emptyset\}$ with compact, convex values and $F_{0}(t, x) \subset F(t, x)$, for all $(t, x) \in[a, b] \times X$, having the following properties:

(a) if $u, v:[a, b] \rightarrow \mathbb{R}^{n}$ are measurable functions with $v(t) \in F(t, u(t))$ on $[a, b]$, then $v(t) \in F_{0}(t, u(t))$ almost everywhere on $[a, b]$;

(b) for every $\varepsilon>0$, there exists a closed $I_{\varepsilon} \subset[a, b]$ such that $\mu\left([a, b] \backslash I_{\varepsilon}\right)<\varepsilon$, $F_{0}(t, x) \neq \emptyset$, for all $(t, x) \in I_{\varepsilon} \times X$ and $F_{0}$ is u.s.c. on $I_{\varepsilon} \times X$.

The proof of main result, Theorem 3.1 below, will be based (except from Proposition 2.1) also on the following proposition developed in [12]. Its proof was based on combination of bound sets approach with the continuation principle developed in [2]. The key point for application of the continuation principle lied in the fact that we assigned to the Dirichlet problem (1.1) the family of associated problems

$$
\left\{\begin{array}{l}
\ddot{x}(t) \in \lambda F(t, q(t), \dot{q}(t)), \quad \text { for a.a. } t \in[0, T], \\
x(T)=x(0)=\mathbf{0},
\end{array}\right.
$$

where $\lambda \in[0,1]$, and

$$
q \in Q:=\left\{q \in C^{1}\left([0, T], \mathbb{R}^{n}\right) \mid q(t) \in \bar{K} \text { for all } t \in[0, T]\right\} .
$$

Proposition 2.2 (cf. [12, Theorem 4.1 and Corollary 3.1]). Let us consider the Dirichlet problem (1.1)-(1.2) where $F:[0, T] \times \mathbb{R}^{n} \times \mathbb{R}^{n} \multimap \mathbb{R}^{n}$ is an upperCarathéodory multivalued mapping. Moreover, assume that

(a) the closure $\bar{K}$ of the set $K$ is a retract of $\mathbb{R}^{n}$,

(b) there exists a nonnegative, integrable function $\beta:[0, T] \rightarrow \mathbb{R}$ such that

$$
|F(t, q(t), \dot{q}(t))| \leq \beta(t), \quad \text { a.e. in }[0, T],
$$

for each $q \in Q$, where $Q$ is defined by (2.2)

(c) there exists a function $V \in C^{2}\left(\mathbb{R}^{n}, \mathbb{R}\right)$ satisfying conditions:

(H1) $\left.V\right|_{\partial K}=0$,

(H2) $V(x) \leq 0$ for all $x \in \bar{K}$,

(d) there exists $\varepsilon>0$ such that, for all $x \in \bar{K} \cap N_{\varepsilon}(\partial K), t \in(0, T), \lambda \in(0,1]$ and $v \in \mathbb{R}^{n}$ with $|v| \leq 2 \int_{0}^{T} \beta(t) d t$, the following condition:

$$
\langle H V(x) v, v\rangle+\langle\nabla V(x), w\rangle>0
$$

holds, for all $w \in \lambda F(t, x, v)$. 
Then the Dirichlet viability problem (1.1)-(1.2) has a solution.

The function $V$ satisfying conditions from Proposition 2.2 is called a (Liapunov-like) bounding function. Its existence guarantees that $K$ is a bound set for the b.v.p.

$$
\left\{\begin{array}{l}
\ddot{x}(t) \in \lambda F(t, x(t), \dot{x}(t)), \text { for a.a. } t \in[0, T], \\
x(T)=x(0)=\mathbf{0},
\end{array}\right.
$$

for all $\lambda \in(0,1]$, i.e. ensures that there does not exist, for any $\lambda \in(0,1]$, a solution $x$ of the b.v.p. (2.4) such that $x(t) \in \bar{K}$, for each $t \in[0, T]$, and $x\left(t_{0}\right) \in \partial K$, for some $t_{0} \in[0, T]$.

\section{Existence and localization result}

Approximating the original problem by a sequence of problems satisfying conditions of Proposition 2.2 and applying the Scorza-Dragoni type result, we are already able to state the main result of the paper. The transversality condition imposed on the bounding function is now required only on the boundary $\partial K$ of the set $K$, and not on the whole neighbourhood $\bar{K} \cap N_{\varepsilon}(\partial K)$, as in Proposition 2.2.

THEOREM 3.1. Let us consider the Dirichlet viability problem (1.1)-(1.2) and assume that

(a) the closure $\bar{K}$ of the set $K$ is a retract of $\mathbb{R}^{n}$,

(b) there exists a nonnegative, integrable function $\alpha:[0, T] \rightarrow \mathbb{R}$ such that

$$
|F(t, q(t), \dot{q}(t))| \leq \alpha(t), \quad \text { a.e. in }[0, T],
$$

for each $q \in Q$, where $Q$ is defined by formula (2.2),

(c) there exists a function $V \in C^{2}\left(\mathbb{R}^{n}, \mathbb{R}\right)$ satisfying conditions $(\mathrm{H} 1)$, (H2),

(d) for all $\lambda \in(0,1], x \in \partial K, t \in(0, T), v \in \mathbb{R}^{n}$ with $|v| \leq 2 \int_{0}^{T}(2 \alpha(t)+1) d t$, and $w \in \lambda F(t, x, v)$, it holds that

$$
\langle\nabla V(x), w\rangle>0
$$

(e) there exists $h>0$ such that $H V(x)$ is positive semi-definite, for all $x \in \bar{K} \cap N_{h}(\partial K)$.

Then the Dirichlet viability problem (1.1)-(1.2) has a solution.

Proof. At first, let us consider the family of associated problems (2.1) and let $x$ be a solution of $(2.1)$ for some $(q, \lambda) \in Q \times(0,1]$. Then it follows from the boundary conditions that there exists a point $\xi \in(0, T)$ such that $\dot{x}(\xi)=0$. Therefore, according to condition (b),

$$
|\dot{x}(0)|=|\dot{x}(\xi)-\dot{x}(0)|=\left|\int_{0}^{\xi} \ddot{x}(t) d t\right| \leq \int_{0}^{\xi}|\ddot{x}(t)| d t \leq \int_{0}^{\xi} \alpha(t) d t \leq \int_{0}^{T} \alpha(t) d t .
$$


Therefore, for almost all $t \in[0, T]$,

$$
|\dot{x}(t)| \leq|\dot{x}(0)|+\int_{0}^{t} \alpha(s) d s \leq 2 \int_{0}^{T} \alpha(s) d s .
$$

Moreover, for almost all $t \in[0, T]$,

$$
|x(t)| \leq|x(0)|+\int_{0}^{t}|\dot{x}(s)| d s \leq 2 \int_{0}^{T} \int_{0}^{T} \alpha(s) d s d u=2 T \int_{0}^{T} \alpha(s) d s .
$$

Thus, $x$ satisfies $|x(t)| \leq a$ and $|\dot{x}(t)| \leq b$, for almost all $t \in[0, T]$, where

$$
a:=2 T \int_{0}^{T} \alpha(s) d s \text { and } b:=2 \int_{0}^{T} \alpha(s) d s .
$$

It follows from condition (d) and from compactness of $\partial K$ that there exists $\delta>0$ such that $\nabla V(x) \neq 0$, for all $x \in N_{\delta}(\partial K)$. Moreover, there exists $\gamma>0$ such that $|\nabla V(x)| \geq \gamma$, for all $x \in \partial K$.

Let us consider an open bounded set $K_{0} \subset \mathbb{R}^{n}$ such that $\bar{K} \subset K_{0}$. Since $F$ is an upper-Carathéodory mapping, we can apply a Scorza-Dragoni type result (cf. Proposition 2.1). Consequently, there exists a decreasing sequence $\left\{\theta_{m}\right\}$ of subsets of $[0, T]$ and a measurable mapping $\bar{F}:[0, T] \times \overline{K_{0}} \times \overline{B_{2 b}} \multimap \mathbb{R}^{n}$ such that, for every $m \in \mathbb{N}$,

- $[0, T] \backslash \theta_{m}$ is compact and $\mu\left(\theta_{m}\right)<1 / m$,

- $\bar{F}(t, x, y) \subset F(t, x, y)$, for all $(t, x, y) \in[0, T] \times \overline{K_{0}} \times \overline{B_{2 b}}$,

- $\bar{F}$ is u.s.c. on $\left([0, T] \backslash \theta_{m}\right) \times \overline{K_{0}} \times \overline{B_{2 b}}$.

It is obvious that $\bigcap_{m=1}^{\infty} \theta_{m}$ has zero Lebesque measure and that $\lim _{m \rightarrow \infty} \chi_{\theta_{m}}(t)=0$, for every $t \notin \bigcap_{m=1}^{\infty} \theta_{m}$. Therefore, $\bar{F}$ is an upper-Carathéodory mapping.

Let us define the mapping $\widehat{F}:[0, T] \times \mathbb{R}^{2 n} \multimap \mathbb{R}^{n}$ by the formula

$$
\widehat{F}(t, x, y):= \begin{cases}\bar{F}(t, x, y), & \text { for }(t, x, y) \in[0, T] \times K_{0} \times B_{2 b}, \\ F(t, x, y), & \text { otherwise. }\end{cases}
$$

Since $K_{0}$ is open and $\hat{F}(t, x, y) \subset F(t, x, y)$, for all $(t, x, y) \in[0, T] \times \mathbb{R}^{2 n}$, the mapping $\widehat{F}$ is also an upper-Carathéodory mapping.

Let $\tau \in C^{1}\left(\mathbb{R}^{n},[0,1]\right)$ be such that $\tau \equiv 1$ on $N_{\delta / 2}(\partial K)$ and $\tau \equiv 0$ on $\mathbb{R}^{n} \backslash N_{\delta}(\partial K)$ and let us consider (for all $m \in \mathbb{N}$ ) the $m$-th problem

$$
\left\{\begin{array}{l}
\ddot{x}(t) \in F_{m}(t, x(t), \dot{x}(t)), \quad \text { for a.a. } t \in[0, T], \\
x(T)=x(0)=\mathbf{0},
\end{array}\right.
$$

where an upper-Carathéodory mapping $F_{m}:[0, T] \times \mathbb{R}^{2 n} \multimap \mathbb{R}^{n}$ is defined by

$$
F_{m}(t, x, y):=F(t, x, y)+\tau(x)\left(\alpha(t) \chi_{\theta_{m}}(t)+\frac{1}{m}\right) \frac{\nabla V(x)}{|\nabla V(x)|} .
$$


Moreover, let us consider the family of problems $\left(\Pi_{m, q, \lambda}\right)$, associated to $\left(\mathrm{P}_{m}\right)$, $\left(\Pi_{m, q, \lambda}\right) \quad\left\{\begin{array}{l}\ddot{x}(t) \in \lambda F_{m}(t, q(t), \dot{q}(t)), \quad \text { for a.a. } t \in[0, T], \\ x(T)=x(0)=\mathbf{0},\end{array}\right.$

where $q \in Q$ and $\lambda \in[0,1]$.

Let us now prove that there exists $m_{0} \in \mathbb{N}$ such that, for all $m \in \mathbb{N}, m \geq m_{0}$, the problem $\left(\mathrm{P}_{m}\right)$ satisfies assumptions (b) and (d) from Proposition 2.2.

(b) Since for all $m \in \mathbb{N}$,

$$
\left|\tau(x)\left(\alpha(t) \chi_{\theta_{m}}(t)+\frac{1}{m}\right) \frac{\nabla V(x)}{|\nabla V(x)|}\right| \leq \alpha(t) \chi_{\theta_{m}}(t)+\frac{1}{m} \leq \alpha(t)+1,
$$

for almost all $t \in[0, T]$, it holds for all $m \in \mathbb{N}, q \in Q$ and almost all $t \in[0, T]$ that

$$
\left|F_{m}(t, q(t), \dot{q}(t))\right| \leq \beta(t),
$$

where $\beta(t):=2 \alpha(t)+1$. Assumption (b) from Proposition 2.2 is therefore satisfied.

(d) If $x_{m}$ be a solution of $\left(\Pi_{m, q, \lambda}\right)$, then

$$
\left|x_{m}(t)\right| \leq a_{m}, \quad\left|\dot{x}_{m}(t)\right| \leq b_{m}, \quad \text { for all } t \in[0, T],
$$

where

$$
a_{m}=2 T \int_{0}^{T}\left(\alpha(s)+\alpha(s) \chi_{\theta_{m}}(s)+\frac{1}{m}\right) d s
$$

and

$$
b_{m}=2 \int_{0}^{T}\left(\alpha(s)+\alpha(s) \chi_{\theta_{m}}(s)+\frac{1}{m}\right) d s .
$$

Since $\mu\left(\theta_{m}\right)<1 / m$ and $1 / m \rightarrow 0$ as $m \rightarrow \infty$, it is easy to see that

$$
\int_{0}^{T}\left(\alpha(s) \chi_{\theta_{m}}(s)+\frac{1}{m}\right) d s \rightarrow 0,
$$

implying $a_{m} \rightarrow a$ and $b_{m} \rightarrow b$ as $m \rightarrow \infty$, where $a$ and $b$ are defined by (3.2). Therefore, it is possible to find $m^{*} \in \mathbb{N}$ such that $a_{m}<2 a$ and $b_{m}<2 b$, for all $m \in \mathbb{N}, m \geq m^{*}$.

Let us now verify condition (d) for all $m \in \mathbb{N}, m \geq m^{*}$.

At first, consider an arbitrary $t \in \theta_{m}, x \in N_{\delta / 2}(\partial K) \cap N_{h}(\partial K) \cap \bar{K}, \lambda \in(0,1]$, $v \in \mathbb{R}^{n}$ with $|v| \leq 2 \int_{0}^{T}(2 \alpha(t)+1) d t$ and $w_{m} \in \lambda F_{m}(t, x, v)$. Then

$$
w_{m}=w+\tau(x)\left(\alpha(t) \chi_{\theta_{m}}(t)+\frac{1}{m}\right) \frac{\nabla V(x)}{|\nabla V(x)|}
$$

with $w \in \lambda \widehat{F}(t, x, v)$ and

$$
\langle H V(x) v, v\rangle+\left\langle\nabla V(x), w_{m}\right\rangle \geq\left\langle\nabla V(x), w_{m}\right\rangle,
$$


by means of condition (e). Moreover,

$$
\begin{aligned}
\left\langle\nabla V(x), w_{m}\right\rangle & =\langle\nabla V(x), w\rangle+\tau(x)\left(\alpha(t) \chi_{\theta_{m}}(t)+\frac{1}{m}\right) \cdot|\nabla V(x)| \\
& =\langle\nabla V(x), w\rangle+\left(\alpha(t)+\frac{1}{m}\right) \cdot|\nabla V(x)| \\
& \geq\left(-|w|+\alpha(t)+\frac{1}{m}\right) \cdot|\nabla V(x)|>0,
\end{aligned}
$$

because $|\nabla V(x)|>0$, and since $|w| \leq \alpha(t)$.

Let $t \in(0, T) \backslash \theta_{m}, x \in \partial K, v \in \mathbb{R}^{n}$ with $|v| \leq 2 \int_{0}^{T}(2 \alpha(t)+1) d t, \lambda \in(0,1]$ and $w_{m} \in \lambda F_{m}(t, x, v)$. Then $\chi_{\theta_{m}}(t)=0, \tau(x)=1$ and

$$
\langle H V(x) v, v\rangle+\left\langle\nabla V(x), w_{m}\right\rangle \geq\left\langle\nabla V(x), w_{m}\right\rangle,
$$

according to condition (e). Moreover, there exists $w \in \lambda \widehat{F}(t, x, v)$ such that

$$
\left\langle\nabla V(x), w_{m}\right\rangle=\langle\nabla V(x), w\rangle+\frac{1}{m} \cdot|\nabla V(x)|>\frac{\gamma}{m},
$$

by means of condition (d) and reasonings at the beginning of the proof.

According to the Scorza-Dragoni result and since $\widehat{F}=\bar{F}$ on $\left([0, T] \backslash \theta_{m}\right) \times \bar{K} \times$ $\overline{B_{2 b}}$, the mapping $\widehat{F}$ is u.s.c. on $\left([0, T] \backslash \theta_{m}\right) \times \bar{K} \times \overline{B_{2 b}}$. Moreover, $V \in C^{2}\left(\mathbb{R}^{n}, \mathbb{R}\right)$, and therefore, there exists $\kappa_{m}>0$ such that

$$
\langle H V(x) v, v\rangle+\left\langle\nabla V(x), w_{m}\right\rangle>0,
$$

for all $t \in(0, T) \backslash \theta_{m}, x \in N_{\kappa_{m}}(\partial K) \cap \bar{K} \cap N_{h}(\partial K), v \in \mathbb{R}^{n}$ with $|v| \leq 2 \int_{0}^{T}(2 \alpha(t)$ $+1) d t, \lambda \in(0,1]$ and $w_{m} \in \lambda F_{m}(t, x, v)$.

Assumption (d) is, therefore, satisfied with $\varepsilon=\min \left\{\delta / 2, \kappa_{m}, h\right\}$.

Thus, we can apply Proposition 2.2 obtaining, for all $m \geq m^{*}$, the existence of a solution $x_{m}$ of the $m$-th problem $\left(\mathrm{P}_{m}\right)$ such that $x_{m}(t) \in \bar{K}$, for each $t \in[0, T]$. Due to the continuation principle (see [2]) used for solving $\left(\mathrm{P}_{m}\right), x_{m}$ is indeed a solution of $\left(\Pi_{m, q, \lambda}\right)$, for $\lambda=1$. Therefore, according to the previous part of this proof, we obtain that $\left|\dot{x}_{m}(t)\right| \leq 2 b$, for all $m \geq m^{*}$ and $t \in[0, T]$, where $b$ is defined by $(3.2)$, and $\left|\ddot{x}_{m}(t)\right| \leq 2 \alpha(t)+1$. It is then possible to get $x \in C^{1}\left([0, T], \mathbb{R}^{n}\right)$ with absolutely continuous $\dot{x}$ and a subsequence (see e.g. [3, Theorem 0.3.4]), again denoted as the sequence, such that $x_{m} \rightarrow x, \dot{x}_{m} \rightarrow \dot{x}$, uniformly in $[0, T]$, and $\ddot{x}_{m} \rightarrow \ddot{x}$, weakly in $L^{1}\left([0, T], \mathbb{R}^{n}\right)$, as $m \rightarrow \infty$. Thus, $x$ satisfies the boundary conditions in (1.1). Put

$$
\varphi_{m}(t):=\tau\left(x_{m}(t)\right)\left(\alpha(t) \chi_{\theta_{m}}(t)+\frac{1}{m}\right) \cdot \frac{\nabla V\left(x_{m}(t)\right)}{\left|\nabla V\left(x_{m}(t)\right)\right|} .
$$

Since $\left|\varphi_{m}(t)\right| \leq \alpha(t) \chi_{\theta_{m}(t)}+1 / m$, for almost all $t \in[0, T]$, and $\varphi_{m}(t) \rightarrow 0$ as $m \rightarrow \infty$ in $[0, T]$, we have that $\left(\dot{x}_{m}(t), \ddot{x}_{m}(t)-\varphi_{m}(t)\right) \rightarrow(\dot{x}, \ddot{x})$, weakly in $L^{1}\left([0, T], \mathbb{R}^{n}\right)$, as $m \rightarrow \infty$. Therefore, a standard limiting argument implies that $x$ is a solution of problem (1.1). Finally, since $x_{m}(t) \in \bar{K}$, for all $m \in \mathbb{N}$ and 
$t \in[0, T]$, we obtain that also $x(t) \in \bar{K}$, for all $t \in[0, T]$, which completes the proof.

REMARK 3.2. As pointed out in the proof, the founded solution $x$ of problem (1.1)-(1.2) is indeed a solution of (2.1), for $q=x$ and $\lambda=1$. Therefore, due to the proof and the assumption (b), $|\dot{x}(t)| \leq b$, for all $t \in[0, T]$, where $b:=$ $2 \int_{0}^{T} \alpha(s) d s$. It is so possible to enlarge the localization conditions and ensure the existence of solution of problem

$$
\begin{aligned}
& \left\{\begin{array}{l}
\ddot{x}(t) \in F(t, x(t), \dot{x}(t)), \quad \text { for a.a. } t \in[0, T], \\
x(T)=x(0)=0,
\end{array}\right. \\
& \qquad \begin{aligned}
x(t) \in K, & \text { for all } t \in[0, T], \\
\dot{x}(t) \in \overline{B_{b}}, & \text { for all } t \in[0, T] .
\end{aligned}
\end{aligned}
$$

As an application of Theorem 3.1, we conclude by the dry friction Dirichlet problem.

Example 3.3. Let us consider the vector dry friction b.v.p.

$$
\left\{\begin{array}{l}
\ddot{x}(t)+a \cdot \operatorname{sgn} \dot{x}(t)=\varphi(t, x(t)), \quad \text { for a.a. } t \in[0, T], \\
x(T)=x(0)=\mathbf{0},
\end{array}\right.
$$

where $a \in \mathbb{R}, x(t)=\left(x_{1}(t), \ldots, x_{n}(t)\right)^{T}, \operatorname{sgn} \dot{x}(t)=\left(\operatorname{sgn} \dot{x}_{1}(t), \ldots, \operatorname{sgn} \dot{x}_{n}(t)\right)^{T}$, and $\varphi:[0, T] \times \mathbb{R}^{n} \rightarrow \mathbb{R}^{n}$ is a Carathéodory function such that

$$
|\varphi(t, x)| \leq \beta(t)(1+|x|) \quad \text { with } \beta \in L^{1}([0, T],[0, \infty)) .
$$

Because of discontinuity in $\operatorname{sgn} y$, we can only consider Filippov solutions which can be identified (see e.g. [1], [4], [8]) as Carathéodory solutions of

$$
\left\{\begin{array}{l}
\ddot{x}(t) \in \varphi(t, x(t))-a \cdot \operatorname{Sgn} \dot{x}(t), \quad \text { for a.a. } t \in[0, T], \\
x(T)=x(0)=\mathbf{0},
\end{array}\right.
$$

where $\operatorname{Sgn} y=\left(\operatorname{Sgn} y_{1}, \ldots, \operatorname{Sgn} y_{n}\right)^{T}$ and, for all $i \in\{1, \ldots, n\}$,

$$
\operatorname{Sgn} y_{i}:= \begin{cases}-1, & \text { for } y_{i}<0 \\ {[-1,1],} & \text { for } y_{i}=0 \\ 1, & \text { for } y_{i}>0\end{cases}
$$

If there exist $D>0$ such that $\langle\varphi(t, x), x\rangle-a \sqrt{D} \sqrt{n}>0$, for all $t \in(0, T)$ and $x \in \mathbb{R}^{n}$ with $|x|=D$, then the dry friction b.v.p. (3.4) admits, according to Theorem 3.1, a solution $x$ such that $|x|<D$.

More concretely, for verifying conditions (a)-(e) from Theorem 3.1, let us define the set $K:=\left\{x \in \mathbb{R}^{n}|| x \mid<D\right\}$, the bounding function $V(x):=$ $\left(|x|^{2}-D^{2}\right) / 2$, and $\alpha(t):=\beta(t)(1+D)$. Then, for all $x \in \mathbb{R}^{n}, \nabla V(x)=x$ and 
$H V(x)=I$, and conditions (a), (c), (e) from Theorem 3.1 are obviously satisfied. Moreover, since for all $\lambda \in(0,1], x \in \partial K, v \in \mathbb{R}^{n}$ and $w \in \lambda \cdot \varphi(t, x)-\lambda \cdot a \cdot \operatorname{Sgn} v$, $\langle\nabla V(x), w\rangle \geq \lambda\left(\langle x, \varphi(t, x)\rangle-a\left(\left|x_{1}\right|+\ldots+\left|x_{n}\right|\right)\right) \geq \lambda(\langle\varphi(t, x), x\rangle-a \sqrt{D} \sqrt{n})>0$, condition (d) from Theorem 3.1 holds, too.

All assumptions of Theorem 3.1 are so satisfied, by which, the dry friction problem (3.4) admits a solution $x$ such that $|x|<D$. This solution represents the Filippov solution of the original problem (3.3).

\section{REFERENCES}

[1] J. Andres and L. Gónniewicz, Topological Fixed Point Principles for Boundary Value Problems, Topological Fixed Point Theory and Its Applications, vol. 1, Kluwer, Dordrecht, 2003.

[2] J. Andres And M. PavlačKovÁ, Asymptotic boundary value problems for second-order differential systems, Nonlinear. Anal. 71, 5-6 (2009), 1462-1473.

[3] J.-P. Aubin and A. Cellina, Differential Inclusions, Springer, Berlin, 1984.

[4] K. Deimling, Multivalued Differential Equations, Walter de Gruyter, Berlin, 1992.

[5] L. Ferracuti, C. Marcelli and F. Papalini, Boundary Value Problems for Highly Nonlinear Inclusions Governed by Non-surjective $\Phi$-Laplacians, Set-Valued Anal. 19, 1 (2011), 1-21.

[6] M. Frigon, Théorèmes d'existence de solutions d'inclusions différentialles, Topological Methods in Differential Equations and Inclusions (A. Granas and M. Frigon, eds.), Kluwer Academic, Dordrecht, 1995, 51-87.

[7] M. KožušníKová, A bounding functions approach to multivalued Dirichlet problem, Atti Semin. Mat. Fis. Univ. Modena Reggio Emilia 55 (2007), 1-19.

[8] J. Kunzweil, Ordinary Differential Equations, Elsevier \& SNTL, Dordrecht, Prag, 1986.

[9] S. Kyritsi, N. Matzakos And N.S. Papageorgiou, Nonlinear boundary value problems for second order differential inclusions, Czechoslovak Math. J. 55 (2005), 545-579.

[10] D. MikLaszewski, The two-point problem for nonlinear ordinary differential equations and differential inclusions, Univ. Iagell Acta Math. 36 (1998), 127-132.

[11] M. Palmucci and F. Papalini, Periodic and boundary value problems for second order differential inclusions, J. Appl. Math. Stoch. Anal. 14 (2001), 161-182.

[12] M. PavlaČKovÁ, A bound sets technique for Dirichlet problem with an upperCarathéodory right-hand side, Acta Univ. Palacki. Olomuc., Fac. Rer. Nat., Mathematica 49, 2 (2010), 95-106.

[13] Q. Zhang and G. Li, Semilinear Second Order Differential Inclusions with Nonlinear Boundary Values, Int. J. Nonlinear Sci. 9, 1 (2010), 84-103.

Martina PaVlaČKová

Department of Math. Analysis and Appl. of Mathematics

Faculty of Science

Palacký University

17. listopadu 12

77146 Olomouc, Czech Republic

E-mail address: martina.pavlackova@upol.cz

TMNA : Volume $44-2014-\mathrm{N}^{\mathrm{O}} 1$ 